THEP $02 / 17$

TTP02-41

hep-ph/0212299

\title{
Towards Order $\alpha_{s}^{4}$ Accuracy in $\tau$-decays
}

\author{
P.A. Baikov \\ Institute of Nuclear Physics, \\ Moscow State University Moscow 119992, Russia \\ K.G. Chetyrkin \\ Fakultät für Physik Albert-Ludwigs-Universität Freiburg, \\ D-79104 Freiburg, Germany \\ J.H. Kühn用 \\ Institut für Theoretische Teilchenphysik, \\ Universität Karlsruhe, D-76128 Karlsruhe, Germany
}

(Dated: November 2, 2018)

\begin{abstract}
Recently computed terms of orders $\mathcal{O}\left(\alpha_{s}^{4} n_{f}^{2}\right)$ in the perturbative series for the $\tau$ decay rate, and similar (new) strange quark mass corrections, are used to discuss the validity of various optimization schemes. The results are then employed to arrive at improved predictions for the complete terms order $\mathcal{O}\left(\alpha_{s}^{4}\right)$ and $\mathcal{O}\left(\alpha_{s}^{5}\right)$ in the massless limit as well as for terms due to the strange quark mass. Phenomenological implications are presented.

PACS numbers: 12.38.Bx, 13.35.Dx, 13.66Bc
\end{abstract}

\section{INTRODUCTION}

The dependence of the $\tau$-decay rate on the strong coupling $\alpha_{s}$ has been used for a determination of $\alpha_{s}$ at lower energies, with the most recent results of $0.334 \pm 0.007_{\exp } \pm 0.021_{\text {theo }}$ and $0.3478 \pm 0.009_{\exp } \pm 0.019_{\text {theo }}$ by the ALEPH [1] and OPAL [2 collaborations. After evolution up to higher energies these results agree remarkably well with determinations based on the hadronic $Z$ decay rate. In view of the relatively large value of $\alpha_{s}\left(M_{\tau}\right)$ estimates for the yet unknown terms of higher orders play an important role in current determinations of $\alpha_{s}$ at low energies. This is in contrast to high energy measurements, where the uncertainty from terms of order $\alpha_{s}^{4}$ of 0.001 to 0.002 [3] is somewhat less or at most comparable to the present experimental errors.

The situation is even more problematic for the determination of the strange quark mass from the Cabbibo suppressed $\tau$ decays. Perturbative QCD corrections affecting the $m_{s}^{2}$ term are extremely large and contributions from increasing powers of $\alpha_{s}$ are barely decreasing, which casts doubts on our ability to extract a reliable result for $m_{s}$ from this (in principle) clean and straightforward measurement औ, 5, 6, 6, 8, 9, 10, 11.

Partial results of order $\alpha_{s}^{4}$ for the absorptive part of the massless vector and scalar correlators have been obtained recently [12], namely terms proportional to $n_{f}^{2}$, where $n_{f}$ denotes the number of massless fermion species. These allow to test two popular optimization schemes - based on the principles of "minimal sensitivity" (PMS) and of fastest apparent convergence (FAC) [13, 14, 15 — which have been used to predict yet uncalulated higher order terms [16, 17].

It will be demonstrated that the predictions of both schemes (coinciding at $\alpha_{s}^{4}$ ) for the coefficient of order $n_{f}^{2} \alpha_{s}^{4}$ are in reasonable agreement with our calculations, which are then used to predict the complete fixed order (FO) and

\footnotetext{
*Electronic address: baikov@theory.sinp.msu.ru

†On leave from Institute for Nuclear Research of the Russian Academy of Sciences, Moscow, 117312, Russia; Electronic address: chet@particle.physik.uni-karlsruhe.de

+Electronic address: Johann.Kuehn@physik.uni-karlsruhe.de
} 
the "contour improved" (CI) 18, 19] $\mathcal{O}\left(\alpha_{s}^{4}\right)$ contributions to the $\tau$ decay rate. Employing the four-loop QCD beta function in combination with improved $\alpha_{s}^{4}$ terms a rough estimate even for $\mathcal{O}\left(\alpha_{s}^{5}\right)$ terms can be obtained. The results lead to fairly stable values for $\alpha_{s}$ consistent with current analysises.

Unfortunately, no essential decrease of the difference between the central values $\alpha_{s}\left(M_{\tau}\right)$ as obtained with FI and CI approaches is observed after including order $\alpha_{s}^{4}$ and $\alpha_{s}^{5}$ corrections, assuming for the moment that these estimates are indeed correct. On the other hand, the theoretical uncertainty assigned to $\alpha_{s}\left(M_{\tau}\right)$ within each method according to standard techniques does decrease significantly.

The implication of this approach for the extraction of $m_{s}$ from Cabbibo suppressed decays will be investigated along the same lines. New results will be presented for the terms of order $n_{f} \alpha_{s}^{3} m_{s}^{2}$ in the total rate. In this case the agreement between FAC/PMS predictions and our results is quite encouraging and naturally suggests the use of the former as a reliable prediction for the complete $\alpha_{s}^{3} m_{s}^{2}$ term. Following an approach discussed in [17], even a rough estimate $m_{s}^{2} \alpha_{s}^{4}$ terms can be obtained from these considerations.

However, the rapid increase of the coefficients indicates that the inherent uncertainty of the present $m_{s}$ determinations will not necessarily decrease with inclusion of the higher orders. As we will see, the situation is somewhat better for the spin one contribution if considered separately.

\section{GENERALITIES}

We start with the well-known representation [20, 21, 22, 23, 24 of the tau-lepton hadronic rate as the contour integral along a circle $\mathrm{C}$ of radius $|s|=M_{\tau}^{2}$

$$
R_{\tau}=6 i \pi \int_{|s|=M_{\tau}^{2}} \frac{d s}{M_{\tau}^{2}}\left(1-\frac{s}{M_{\tau}^{2}}\right)^{2}\left[\Pi^{[2]}(s)-\frac{2}{M_{\tau}^{2}} \Pi^{[1]}(s)\right] .
$$

Here $\Pi^{[1]}$ and $\Pi^{[2]}$ are proper flavour combinations of the polarization operators appearing in the decomposition of the correlators of vector and axial vector currents of light quarks

$$
\begin{aligned}
\Pi_{\mu \nu, i j}^{V / A}\left(q, m_{i}, m_{j}, m, \mu, \alpha_{s}\right) & =i \int d x e^{i q x}\left\langle T\left[j_{\mu, i j}^{V / A}(x)\left(j_{\nu, i j}^{V / A}\right)^{\dagger}(0)\right]\right\rangle \\
& =g_{\mu \nu} \Pi_{i j, V / A}^{[1]}\left(q^{2}\right)+q_{\mu} q_{\nu} \Pi_{i j, V / A}^{[2]}\left(q^{2}\right)
\end{aligned}
$$

with $m^{2}=\sum_{f=u, d, s} m_{f}^{2}$ and $j_{\mu, i j}^{V / A}=\bar{q}_{i} \gamma_{\mu}\left(\gamma_{5}\right) q_{j}$. The two (generically different) quarks with masses $m_{i}$ and $m_{j}$ are denoted by $q_{i}$ and $q_{j}$ respectively.

For the case of the $\tau$-lepton the relevant combinations of quark flavours are $i j=u d$ and $i j=u s$. The polarization functions $\Pi_{V / A}^{[l]}, \quad l=1,2$ are conveniently represented in the form $\left(Q^{2}=-q^{2}\right)$

$$
\left(Q^{2}\right)^{(l-2)} \Pi_{u s, V / A}^{[l]}\left(q^{2}\right)=\frac{3}{16 \pi^{2}} \Pi_{V / A, 0}^{[l]}\left(\frac{\mu^{2}}{Q^{2}}, \alpha_{s}\right)+\frac{3}{16 \pi^{2}} \sum_{D \geq 2} Q^{-D} \Pi_{V / A, D}^{[l]}\left(\frac{\mu^{2}}{Q^{2}}, m_{s}^{2}, \alpha_{s}\right) .
$$

Here the first term on the rhs corresponds to the massless limit while the first term in the sum stands for quadratic mass corrections. We neglect the masses of $u$ and $d$ quarks. Therefore in perturbative QCD $\Pi_{V}^{[l]}=\Pi_{A}^{[l]}$ and we will often omit the subscript $V / A$ in the following. Current conservation implies: $\Pi_{0}^{[1]}=\Pi_{0}^{[2]}$.

The full tau-lepton hadron rate $R_{\tau}$ can be presented as a sum of spin 1 and spin 0 parts, viz.

$$
\begin{aligned}
& R_{\tau}^{(1)}=6 i \pi \int_{|s|=M_{\tau}^{2}} \frac{d s}{M_{\tau}^{2}}\left(1-\frac{s}{M_{\tau}^{2}}\right)^{2}\left[\left(1+2 \frac{s}{M_{\tau}^{2}}\right) \Pi^{(1)}(s)+\Pi^{[1]}(0) / s\right] \\
& R_{\tau}^{(0)}=6 i \pi \int_{|s|=M_{\tau}^{2}} \frac{d s}{M_{\tau}^{2}}\left(1-\frac{s}{M_{\tau}^{2}}\right)^{2}\left[\Pi^{(0)}(s)-\Pi^{[1]}(0) / s\right] .
\end{aligned}
$$

where

$$
\Pi^{(1)}=-\Pi^{[1]} / q^{2}, \quad \Pi^{(0)}=\Pi^{[2]}+\Pi^{[1]} / q^{2} .
$$

and the contribution of the singularity at the origin (proportional to $\Pi^{[1]}(0)$ ) has to be included. A nonvanishing value of $\Pi^{[1]}(0)$ is a nonperturbative constant. 
On the other hand, the unknown constant drops out if one considers moments

$$
R_{\tau}^{(1,0) k, l}\left(s_{0}\right)=\int_{0}^{s_{0}} \frac{d s}{M_{\tau}^{2}}\left(1-\frac{s}{M_{\tau}^{2}}\right)^{k}\left(\frac{s}{M_{\tau}^{2}}\right)^{l} \frac{d R_{\tau}^{(1,0)}}{d s},
$$

with $k \geq 0, \quad l \geq 1$. (Note that the moments introduced in 19 are related to ours as $R_{\tau}^{k l}=R_{\tau}^{(1) k, l}+R_{\tau}^{(0) k, l}$.)

The decay rate $R_{\tau}$ may be expressed as the sum of different contributions corresponding to Cabibbo suppressed or allowed decay modes, vector or axial vector contributions and the mass dimension of the corrections

$$
R_{\tau}=R_{\tau, V}+R_{\tau, A}+R_{\tau, S}
$$

with

$$
\begin{aligned}
& R_{V}=\frac{3}{2}\left|V_{u d}\right|^{2}\left(1+\delta_{0}+\sum_{D=2,4, \ldots} \delta_{V, u d, D}\right), \\
& R_{A}=\frac{3}{2}\left|V_{u d}\right|^{2}\left(1+\delta_{0}+\sum_{D=2,4, \ldots} \delta_{A, u d, D}\right), \\
& R_{S}=3\left|V_{u s}\right|^{2}\left(1+\delta_{0}+\sum_{D=2,4, \ldots} \delta_{u s, D}\right) .
\end{aligned}
$$

Here $D$ indicates the mass dimension of the fractional corrections $\delta_{V / A, i j, D}$, and $\delta_{i j, D}$ denotes the average of the vector and the axial vector contributions: $\delta_{i j, D}=\left(\delta_{V, i j, D}+\delta_{A, i j, D}\right) / 2$. If a decomposition into different spin/parity contributions is made or a particular pattern of moments is considered then we will use the corresponding obvious generalization of (8). For instance,

$$
R_{S, V}^{(1) k l}=a_{k l}\left|V_{u s}\right|^{2}\left(1+\delta_{0}^{k l}+\sum_{D=2,4, \ldots} \delta_{V, u s, D}^{(1), k l}\right)
$$

and

$$
R_{S, V}^{(0) k l}=\left|V_{u s}\right|^{2}\left(\sum_{D=2,4, \ldots} \delta_{V, u s, D}^{(0), k l}\right)
$$

Thus, in our notation we have the relation

$$
\delta_{V, u s, 2}^{k l}=a_{k l} \delta_{V, u s, 2}^{(1), k l}+\delta_{V, u s, 2}^{(0), k l}
$$

The integral in eq. (11) is, obviously, insensitive, to the $Q^{2}$-independent terms in the polarization functions $\Pi_{0}^{[1]}$ and $\Pi_{2}^{[1]}$. This means that without loss of generality we may deal with the corresponding (Adler) $D$-functions, viz.

$$
D_{0}^{[1]}\left(Q^{2}\right) \equiv-\frac{3}{4} Q^{2} \frac{\mathrm{d}}{\mathrm{d} Q^{2}} \Pi_{0}^{[1]}, \quad D_{2}^{[1]}\left(Q^{2}\right) \equiv-\frac{1}{2} Q^{2} \frac{\mathrm{d}}{\mathrm{d} Q^{2}} \Pi_{2}^{[1]} .
$$

An important property of the functions $D_{0}^{[1]}, D_{2}^{[1]}$ and $\Pi_{2}^{[2]}$ is their scale independence, which implies that they are directly related to measurements.

The Adler functions $D_{0}^{[1]}$ and $D_{2}^{[1]}$ have been calculated with $\mathcal{O}\left(\alpha_{s}^{3}\right)$ accuracy, the polarization function $\Pi_{2}^{[2]}$, however, to $\mathcal{O}\left(\alpha_{s}^{2}\right)$ only (see [7] and references therein).

The (apparent) convergence of the perturbative series for $D_{0}^{[1]}$ is acceptable, the one for $D_{2}^{[1]}$ and $\Pi_{2}^{[2]}$ is at best marginal. This has led to significant theoretical uncertainties in extracting $\alpha_{s}$ and to a fairly unstable behaviour in extraction of $m_{s}$ from $\tau$-decays.

To improve the situation, we have computed the two leading terms in the large $n_{f}$ expansion of the next order, i.e. terms of order $\mathcal{O}\left(n_{f}^{3} \alpha_{s}^{4}\right), \mathcal{O}\left(n_{f}^{2} \alpha_{s}^{4}\right)$ to $D_{0}^{[1]}, D_{2}^{[1]}$ and $\mathcal{O}\left(n_{f}^{2} \alpha_{s}^{3}\right), \mathcal{O}\left(n_{f} \alpha_{s}^{3}\right)$ to $\Pi_{2}^{[2]}$. Our results are described in the following section. 


\section{FIXED ORDER RESULTS IN $\alpha_{s}^{3}$ AND $\alpha_{s}^{4}$}

Using the technique described in [12, 25, 26] and the parallel version of FORM [27, 28], the leading and subleading (in $n_{f}$ ) terms of the next order in the perturbative series for $D_{0}^{[1]}, D_{2}^{[1]}$ and $\Pi_{2}^{[2]}$ have been obtained in the standard $\overline{\mathrm{MS}}$ renormalization scheme 29, 30]:

$$
\begin{aligned}
& D_{0}^{[1]}=1+a_{s}+a_{s}^{2}\left\{\left[-\frac{11}{12}+\frac{2}{3} \zeta_{3}\right] n_{f}+\frac{365}{24}-11 \zeta_{3}\right\} \\
& +a_{s}^{3}\left\{\left[\frac{151}{162}-\frac{19}{27} \zeta_{3}\right] n_{f}^{2}+\left[-\frac{7847}{216}+\frac{262}{9} \zeta_{3}-\frac{25}{9} \zeta_{5}\right] n_{f}\right. \\
& \left.+\frac{87029}{288}-\frac{1103}{4} \zeta_{3}+\frac{275}{6} \zeta_{5}\right\} \\
& +a_{s}^{4}\left\{\left[-\frac{6131}{5832}+\frac{203}{324} \zeta_{3}+\frac{5}{18} \zeta_{5}\right] n_{f}^{3}+\left[\frac{1045381}{15552}+\frac{5}{6} \zeta_{3}^{2}\right.\right. \\
& \left.\left.-\frac{40655}{864} \zeta_{3}-\frac{260}{27} \zeta_{5}\right] n_{f}^{2}+d_{0,1}^{[1] 4} n_{f}+d_{0,0}^{[1] 4}\right\} \\
& =1+a_{s}+a_{s}^{2}\left\{-0.1153 n_{f}+1.986\right\} \\
& +a_{s}^{3}\left\{0.08621 n_{f}^{2}-4.216 n_{f}+18.24\right\} \\
& +a_{s}^{4}\left\{-0.01009 n_{f}^{3}+1.875 n_{f}^{2}+d_{0,1}^{[1] 4} n_{f}+d_{0,0}^{[1] 4}\right\}, \\
& D_{2}^{[1]}=m_{s}^{2}\left(1+\frac{5}{3} a_{s}+a_{s}^{2}\left\{\left[-\frac{11}{8}+\frac{2}{3} \zeta_{3}\right] n_{f}+\frac{5185}{144}-\frac{39}{2} \zeta_{3}\right\}\right. \\
& +a_{s}^{3}\left\{\left[\frac{8671}{11664}-\frac{13}{27} \zeta_{3}\right] n_{f}^{2}+\left[-\frac{44273}{972}+\frac{3257}{81} \zeta_{3}\right.\right. \\
& \left.\left.-\frac{5}{6} \zeta_{4}-\frac{1265}{81} \zeta_{5}\right] n_{f}+\frac{2641517}{5184}-\frac{131275}{216} \zeta_{3}+\frac{12845}{36} \zeta_{5}\right\} \\
& +a_{s}^{4}\left\{\left[-\frac{396781}{559872}+\frac{461}{1296} \zeta_{3}-\frac{1}{48} \zeta_{4}+\frac{5}{18} \zeta_{5}\right] n_{f}^{3}+\left[\frac{61913567}{1119744}-\frac{59}{54} \zeta_{3}^{2}\right.\right. \\
& \left.\left.\left.-\frac{352549}{7776} \zeta_{3}+\frac{67}{96} \zeta_{4}+\frac{22859}{3888} \zeta_{5}\right] n_{f}^{2}+d_{2,1}^{[1] 4} n_{f}+d_{2,0}^{[1] 4}\right\}\right) \\
& =m_{s}^{2}\left(1+1.667 a_{s}+a_{s}^{2}\left\{-0.5736 n_{f}+12.57\right\}\right. \\
& +a_{s}^{3}\left\{0.1646 n_{f}^{2}-14.31 n_{f}+149 .\right\} \\
& \left.+a_{s}^{4}\left\{-0.01563 n_{f}^{3}+6.067 n_{f}^{2}+d_{2,1}^{[1] 4} n_{f}+d_{2,0}^{[1] 4}\right\}\right)
\end{aligned}
$$




$$
\begin{aligned}
\Pi_{2}^{[2]}=-4 m_{s}^{2}(1+ & \frac{7}{3} a_{s}+a_{s}^{2}\left\{\left[-\frac{25}{24}-\frac{2}{9} \zeta_{3}\right] n_{f}+\frac{15331}{432}+\frac{359}{54} \zeta_{3}-\frac{520}{27} \zeta_{5}\right\} \\
& +a_{s}^{3}\left\{\left[\frac{2131}{11664}+\frac{19}{81} \zeta_{3}\right] n_{f}^{2}+\left[-\frac{68135}{1944}-\frac{52}{27} \zeta_{3}^{2}\right.\right. \\
\left.\left.\left.-\frac{3997}{486} \zeta_{3}-\frac{5}{6} \zeta_{4}+\frac{3875}{243} \zeta_{5}\right] n_{f}+k_{2,0}^{[2] 3}\right\}\right) & \\
=-4 m_{s}^{2}\left(\begin{array}{r}
1+ \\
+
\end{array}\right. & 2.333 a_{s}+a_{s}^{2}\left\{-1.309 n_{f}+23.51\right\} \\
& \left.+a_{s}^{3}\left\{0.4647 n_{f}^{2}-32.08 n_{f}+k_{2,0}^{[2] 3}\right\}\right)
\end{aligned}
$$

Here we have used $a_{s}=\frac{\alpha_{s}\left(Q^{2}\right)}{\pi}, m_{s}=m_{s}\left(Q^{2}\right)$ and set the normalization scale $\mu^{2}=Q^{2}$; results for generic values of $\mu$ can be easily recovered with the standard renormalization group techniques. The result for the $\alpha_{s}^{4}$ terms in $D_{0}^{[1]}$ has been already presented in 12 , the coefficients of the $\alpha_{s}^{4}$ and $\alpha_{s}^{3}$ terms in in $D_{2}^{[1]}$ and $\Pi_{2}^{[2]}$ respectively are new.

\section{IMPLICATIONS FOR THE $\alpha_{s}^{4}$ PREDICTIONS AND PHENOMENOLOGICAL ANALYSIS}

\section{A. Massless case}

FAC (Fastest Apparent Convergence) and PMS (Principle of Minimal Sensitivity) methods are both based eventually on the concept of scheme-invariant properties and the idea of the choice of an "optimal" scheme to provide better convergence of the resulting perturbative series. For both methods the optimal scheme depends on the physical observable we are dealing with. With FAC it should be a scheme which minimizes (set to zero by construction) all the terms of order $\alpha_{s}^{2}$ and higher, while the PMS scheme is fixed by the requirement that the perturbative expansion for the observable is as insensitive as possible to a change in the scheme. The assumption that a renormalization scheme is in a sense optimal sets certain constraints on not yet computed higher order corrections in any other scheme. These constraints can be used to "predict" (at least roughly) the magnitude of these corrections.

For the function $D_{0}^{[1]}$ the result is known since long from Ref. [16] (see Table 1, column 5). From the three entries corresponding to $n_{f}=3,4$ and 5 one easily restores the FAC/PMS prediction for the $n_{f}$ dependence of the $\alpha_{s}^{4}$ term in $D_{0}^{[1]}$ (the term of order $n_{f}^{3}$ was fixed to its computed value):

$$
d_{0}^{[1] 4}=127.6-44.2 n_{f}+3.64 n_{f}^{2}-0.0100928 n_{f}^{3} d_{0}^{[1] 4}=127.58-44.211 n_{f}+3.6439 n_{f}^{2}-0.0100928 n_{f}^{3} .
$$

(Note that FAC and PMS predictions happen to coincide for the $\alpha_{s}^{4}$ term.)

\begin{tabular}{|c|c|c|c|c|c|}
\hline$n_{f}$ & $d_{3}^{\text {exact }}$ & $d_{3}^{\text {FAC }}$ & $d_{3}^{\text {PMS }}$ & $d_{4}^{\text {FAC } / \text { PMS }}$ & $d_{5}^{\text {FAC }}$ \\
\hline \hline 3 & 6.371 & 5.604 & 6.39 & $27 \pm 16$ & $145 \pm 100$ \\
\hline 4 & 2.758 & 4.671 & 5.26 & $8 \pm 28$ & $40 \pm 160$ \\
\hline 5 & -0.68 & 3.762 & 4.16 & $-8 \pm 44$ & $-3 \pm 230$ \\
\hline
\end{tabular}

TABLE I: Estimates for the coefficients $d_{3}=d_{0}^{[1] 3}$ and $d_{4}=d_{0}^{[1] 4}$ in the function $D_{0}^{[1]}$ based on FAC and PMS optimizations. The estimate for of $d_{0}^{[1] 4}$ employs the exact value of $d_{0}^{[1] 3}$. The last column contains the FAC predictions for the coefficient $d_{5}^{\mathrm{FAC}}$ which was obtained assuming the value for $d_{0}^{[1] 4}$ as given in the fifth column; the corresponding uncertainties have been estimated as described in the text.

It is interesting to compare the FAC/PMS predictions for the $n_{f}$ dependence of coefficient $d_{0}^{[1] 3}$ with the exact result given in eq. 12. The results of both estimates are

$$
d_{0}^{[1] 3}(F A C)=8.54-1.013 n_{f}+0.0116 n_{f}^{2},
$$




$$
d_{0}^{[1] 3}(P M S)=9.93-1.23 n_{f}+0.0125 n_{f}^{2} .
$$

The comparison of the complete $\alpha_{s}^{3}$ and partial $\alpha_{s}^{4}$ results with FAC and PMS estimates leads to the following observations:

- Starting from $\alpha_{s}^{3}$, the leading in $\alpha_{s}$ and $n_{f}$ terms of order $\alpha_{s}^{3} n_{f}^{2}$ and $\alpha_{s}^{4} n_{f}^{3}$ are numerically quite small (at least for $\left.n_{f} \leq 6\right)$ and, thus, should have a negligibly small influence on the coefficients of the $\alpha_{s}$ expansion. On the other hand, the term subleading in $n_{f}$, say, of order $\alpha_{s}^{3} n_{f}$ is comparable in size with the term of order $\alpha_{s}^{3} n_{f}^{0}$. Similarly, the $\alpha_{s}^{4} n_{f}^{3}$ term is significantly smaller than the $\alpha_{s}^{4} n_{f}^{2}$ one, whereas the $\alpha_{s}^{4} n_{f}$ and $\alpha_{s}^{4} n_{f}^{0}$ terms are expected to be of similar magnitude.

- In general FAC/PMS methods correctly reproduce sign and order of magnitude of the higher order coefficients. The agreement is getting better when the coefficients happen to be large, as is the case for the quadratic mass corrections (see below).

- Taken separately, the FAC/PMS estimates of the coefficients of the $n_{f}$ expansion could deviate rather strongly from the true result. However, for a given $n_{f}$, the deviation in the predicted value of the full $O\left(\alpha_{s}^{n}\right)$ term tends to be significantly smaller than what could be expected from summing individual terms of the $n_{f}$ expansion. In addition, for the particular point $n_{f}=3$ very good agreement is observed.

To illustrate this feature, let us consider the worst case: the FAC/PMS prediction for the $\alpha_{s}^{3} n_{f}$ term in the function $d_{0}^{[1]}$. Here the ratio of the exact result relative to the predicted one is quite large (about 4). Without knowledge of the $\alpha_{s}^{3} n_{f}^{0}$ contribution one would expect that the uncertainty of the prediction for the full $\alpha_{s}^{3}$ coefficient should be at least around

$$
\left(\left.d_{0,1}^{[1] 3}\right|_{\text {exact }}-\left.d_{0,1}^{[1] 3}\right|_{F A C}\right) n_{f} .
$$

For $n_{f}=3,4,5$ this amounts to 9,12 and 15 , which should be compared to the corresponding differences of the full order $O\left(\alpha_{s}^{3}\right)$ (summed over all contributing power of $n_{f}$ ) coefficients, viz. 1,2,4. This example demonstrates that the deviation of FAC/PMS predictions for subleading in $n_{f}$ terms may well serve as conservative estimate of the accuracy in the prediction of the complete terms of orders $\alpha_{s}^{3}$ and $\alpha_{s}^{4}$.

These observations motivate the assumption that the prediction for the coefficient $d_{0}^{[1] 4}\left(\equiv d_{0,0}^{[1] 4}+d_{0,1}^{[1] 4} n_{f}+d_{0,2}^{[1] 4} n_{f}^{2}+\right.$ $\left.d_{0,3}^{[1] 4} n_{f}^{3}\right)$ should also be correct within

$$
\pm\left(\left.d_{0}^{[1] 4}\right|_{\text {exact }}-\left.d_{0}^{[1] 4}\right|_{F A C}\right) n_{f}^{2}
$$

Thus, in our phenomenological analysis of the $\tau$-lepton decays the estimate

$$
\left.d_{0}^{[1] 4}\right|_{n_{f}=3}=27 \pm 16
$$

will be used. On the basis of this improved estimate and the four loop $\beta$ function 31] one may even speculate about the $\alpha_{s}^{5}$ term (whose exact evaluation is completely out of reach in the foreseeable future). Following the discussion of Kataev and Starshenko [17], one obtains

$$
\left.d_{0}^{[1] 5}\right|_{n_{f}=3}=145 \pm 100,
$$

not far from the previous estimates of Ref. 17]. The variation of $d_{0}^{[1] 4}$ by \pm 16 leads to the variation of $d_{0}^{[1] 5}$ by \pm 100 . For other values of $n_{f}$, the corresponding predictions can be obtained in the same way. They are listed in Table 1.

The FAC/PMS prediction (15) for the $n_{f}$ dependence of the coefficient $d_{0}^{[1] 4}$ does not take into account the available knowledge of the corresponding $n_{f}^{2}$ part. One can easily include this by fitting the FAC/PMS predictions for only two values of $n_{f}$ with a linear function of $n_{f}$. As a result one obtains 38 (we have boxed the predicted coefficients in order to separate them clearly from the input)

$$
\begin{aligned}
& d_{0}^{[1] 4}\left(\mathrm{FAC} / \mathrm{PMS}, n_{f}=3,4\right)=105.7-31.8 n_{f}+1.875 n_{f}^{2}-0.01009 n_{f}^{3}, \\
& d_{0}^{[1] 4}\left(\mathrm{FAC} / \mathrm{PMS}, n_{f}=4,5\right)=107.7-32.3 n_{f}+1.875 n_{f}^{2}-0.01009 n_{f}^{3}, \\
& d_{0}^{[1] 4}\left(\mathrm{FAC} / \mathrm{PMS}, n_{f}=3,5\right)=106.4-32.0 n_{f}+1.875 n_{f}^{2}-0.01009 n_{f}^{3} .
\end{aligned}
$$


One could now perform a self-consistency check of Table I by predicting, say, $d_{0}^{[1] 4}$ for $n_{f}=5$ from eq. (20). The result - $(-7.5)$ - is compared successfully to the value listed in the table, viz. - 8 . The corresponding predictions from eqs. (21) and (22) are also in very good agreement to Table I.

An instructive example of how knowledge and inclusion of the subleading $n_{f}$ term can improve FAC/PMS predictions is provided by the (exactly known) coefficient $d_{0}^{[1] 3}$. Indeed, assuming the knowledge of $d_{0,2}^{[1] 3}$ and $d_{0,1}^{[1] 3}$ and using the values of $d_{3}^{\text {FAC }}$ and $d_{3}^{\text {PMS }}\left(\right.$ at $n_{f}=3$ ) as given by Table 1 , one easily arrives at

$$
d_{0}^{[1] 3}(\mathrm{FAC})=17.48-4.216 n_{f}+0.08621 n_{f}^{2}
$$

and

$$
d_{0}^{[1] 3}(\mathrm{PMS})=18.27-4.216 n_{f}+0.08621 n_{f}^{2},
$$

which should be compared to the exact value $d_{0,0}^{[1] 3}=18.24$. Repeating the same exercise for $n_{f}=4,5$ we get 20.16 (FAC) and 20.75 (PMS) for $n_{f}=4$ as well as 22.69 (FAC) and 23.08 (PMS) for $n_{f}=5$ respectively.

Bearing in mind that in many cases FAC/PMS predictions made for $n_{f}=3$ are in better agreement with the exact results (see, e.g. Table I and tables from Ref. [17]), we suggest eq. (20) as the best FAC/PMS prediction for the constant term and the term linear in $n_{f}$ in the coefficient $d_{0}^{[1] 4}$.

Eqs. (18,19) can be used to predict $R_{\tau}$ in the massless limit first in fixed order perturbation theory (FOPT)

$$
\begin{gathered}
R_{\tau}^{\mathrm{FOPT}}= \\
3\left(1+a_{s}+5.202 a_{s}^{2}+26.37 a_{s}^{3}+a_{s}^{4}(105 \pm 16)+a_{s}^{5}(138 \pm 230 \pm 100)\right) .
\end{gathered}
$$

Here $a_{s}=\alpha_{s}\left(M_{\tau}\right) / \pi$. The first uncertainty in the $\alpha_{s}^{5}$ term comes from that of $d_{0}^{[1] 4}$ while the second is our estimation of the error in the very coefficient $d_{0}^{[1] 5}$. (Of course, within this approach they are strongly correlated).

Similarly, we can use "contour improved" (CI) formulae [18, 19] (assuming as reference value $\alpha_{s}\left(M_{\tau}\right)=0.334$ [1]) to get

$$
R_{\tau}^{C I}=3\left(1+1.364 a_{s}+2.54 a_{s}^{2}+9.71 a_{s}^{3}+1.31 a_{s}^{4} d_{0}^{[1] 4}+0.95 a_{s}^{5} d_{0}^{[1] 5}\right)
$$

or, equivalently,

$$
R_{\tau}^{C I}=3\left(1+1.364 a_{s}+2.54 a_{s}^{2}+9.71 a_{s}^{3}+a_{s}^{4}(35 \pm 20)+a_{s}^{5}(138 \pm 95)\right)
$$

Let us compare our new value for the coefficient $d_{0}^{[1] 4}$ in (18) with the ones used in extracting $\alpha_{s}\left(M_{\tau}\right)$ from $\tau$ data by the OPAL [2] and ALEPH [1] collaborations, namely

$$
\left.d_{0}^{[1] 4}\right|_{n_{f}=3}=25 \pm 25(\mathrm{OPAL}), \quad 50 \pm 50(\mathrm{ALEPH}) .
$$

While OPAL's central value is basically the same as ours, their error bar is somewhat larger. In the case of ALEPH both the central value and the uncertainty assigned to it are significantly larger than our numbers. In this connection, we would like to stress that eq. (18) utilizes completely new non-trivial information given in (12): the subleading term in $n_{f}$ term of order $\alpha_{s}^{4}$.

It is of interest to see in detail which accuracy in the determination of $\alpha_{s}\left(M_{\tau}\right)$ one could achieve assuming eqs. $(18,19)$. Let us introduce the quality $\delta_{P}$ as follows:

$$
R_{\tau S=0}=\frac{\Gamma\left(\tau \rightarrow h_{S=0} \nu\right)}{\Gamma(\tau \rightarrow l \bar{\nu} \nu)}=\left|V_{u d}\right|^{2} S_{E W} R_{\tau},
$$

with

$$
R_{\tau}=3\left(1+\delta_{P}+\delta_{E W}+\delta_{N P}\right) .
$$

The first term here is the parton result, the second stands for pQCD effects. The non-perturbative corrections represented by $\delta_{N P}$ happens to be rather small $\delta_{N P}=-.003 \pm 0.003$ (see, e.g. [22]). Here the flavour mixing matrix element $\left|V_{u d}\right|^{2}=0.9475 \pm 0.0016$ [32]. The factor $S_{E W}=1.0194$ is the electroweak correction which collects the large logarithmic terms [33], while $\delta_{E W}=0.001$ is an additive electroweak correction [34]. Using for definiteness the result of ALEPH [1]

$$
R_{\tau S=0}=3.492 \pm 0.016
$$


one arrives at

$$
\delta_{P}^{\exp }=0.207 \pm 0.007
$$

To get a value for $\alpha_{s}\left(M_{\tau}\right)$ one should simply fit $\delta_{P}^{\text {exp }}$ against $R_{\tau} / 3-1$ as given by eq. (25) or by eq. (27) to get a result corresponding FOPT or CIPT (Fixed Order or Contour Improved PT).

Unfortunately, there is no unique way to assign a theoretical uncertainty $\delta \alpha_{s}$ to the obtained value of $\alpha_{s}\left(M_{\tau}\right)$. In the literature one finds several suggestions. Let us consider them in turn.

1. $\delta \alpha_{s}$ is a half of the shift in $\alpha_{s}$ induced by the last fully computed term in the PT (that is by the one of order $\alpha_{s}^{3}$ at present).

2. $\delta \alpha_{s}$ is equal to the change in $\alpha_{s}$ caused by varying the normalization point $\mu$ around $M_{\tau}$, typically within the range of $1.1-2.5 \mathrm{GeV}$.

The corresponding results read (terms of order $\alpha_{s}^{4}$ and higher in eq. 25) and in the $D$-function have been set to zero)

$$
\begin{aligned}
\alpha_{s}^{\mathrm{FOPT}}\left(M_{\tau}\right) & =0.345 \pm(0.025 \mid 0.037) \\
\alpha_{s}^{\mathrm{CIPT}}\left(M_{\tau}\right) & =0.364 \pm(0.012 \mid 0.021)
\end{aligned}
$$

Here the first(second) value in brackets correspond to the use of the first(second) suggestion for the error estimation. After evolution from $M_{\tau}$ to $M_{Z}$ this corresponds to

$$
\begin{aligned}
\alpha_{s}^{\mathrm{FOPT}}\left(M_{Z}\right) & =0.1209 \pm(0.0024 \mid 0.0037) \\
\alpha_{s}^{\mathrm{CIPT}}\left(M_{Z}\right) & =0.1229 \pm(0.0011 \mid 0.0020) .
\end{aligned}
$$

3. $\delta \alpha_{s}$ is equal to the change in $\alpha_{s}$ caused by the uncertainty in the predicted (that is not yet completely known) higher order terms in the perturbative series for $R_{\tau}$;

4. $\delta \alpha_{s}$ is a half of the difference in the $\alpha_{s}\left(M_{\tau}\right)$ as obtained within FOPT and CIPT. This difference comes from different handling of higher order terms.

In order to quantify the error estimates according to 3 . and 4 . we have shown in Table II the results for $\alpha_{s}\left(M_{\tau}\right)$ obtained with various choices for $d_{0}^{[1] 4}, d_{0}^{[1] 5}$ and $\mu$. The entries with the choices \pm 100 for the coefficients illustrate the large change in $\alpha_{s}$ which would result from a failure of PMS and FAC once higher order terms are included. For the plausible values of $d_{0}^{[1] 4}$ and $d_{0}^{[1] 5}$ we observe an significant decrease of the $\mu$ dependence after inclusion of additional terms in the $\alpha_{s}$ series.

Our final predictions for $\alpha_{s}^{\mathrm{FOPT}}\left(M_{\tau}\right)$ and $\alpha_{s}^{\mathrm{CIPT}}\left(M_{\tau}\right)$ are given in the first column of Table III, together with experimental error 39 and the combined theory uncertainty. The values of theory uncertainties are listed separately in columns 3,4 and 5 . The corresponding values at the scale of $M_{Z}$ are

$$
\begin{aligned}
\alpha_{s}^{\mathrm{FOPT}}\left(M_{Z}\right) & =0.1192 \pm 0.0007 \pm 0.002 \\
\alpha_{s}^{\mathrm{CIPT}}\left(M_{Z}\right) & =0.1219 \pm 0.001 \pm 0.0006
\end{aligned}
$$

Thus we observe that the total uncertainty based on a combination of not yet calculated higher order terms, $\mu$ dependence and scheme dependence is reduced, once $\alpha_{s}^{4}$ terms are available. However, the difference between FOPT and CIPT results of roughly 0.02 is a remaining, at the moment irreducible uncertainty 40 .

It is thus of interest to study this difference as a function of $m_{\tau}$. In practice, this could be applied to sum rules for spectral functions as determined in $e^{+} e^{-}$annihilation.

Therefore, let us consider a hypothetical case of $\tau$ lepton with the mass equal $3 \mathrm{GeV}$. Assuming $\alpha_{s}(1.77 \mathrm{GeV})=0.334$ and running this value to $3 \mathrm{GeV}$ via standard 4-loop evolution equation one gets $\alpha_{s}(3 \mathrm{GeV})=0.2558$ and predicts

$$
\delta_{P}^{\exp }=0.1353
$$

which corresponds to eq. (25) with the $\alpha_{s}^{4}$ and $\alpha_{s}^{5}$ terms fixed to their central values (see eqs. (18 19). Let us now investigate the results for $\alpha_{s}$ and the theory error that would result from $\delta_{P}^{\exp }=0.1353$ as a starting point. The corresponding analogs of eqs. (32 35) and Table (II) are displayed below as eqs. ((38),39) and Table (IV) 


\begin{tabular}{|c|c|c|c|}
\hline$d_{0}^{[1] 4}$ & $\alpha_{s}^{4}$ & $d_{0}^{[1] 5}$ & $\alpha_{s}^{5}$ \\
\hline \multirow[t]{2}{*}{27} & $0.331 \pm 0.02$ & \multirow[t]{2}{*}{145} & $0.33 \pm 0.02$ \\
\hline & $0.357 \pm 0.009$ & & $0.354 \pm 0.004$ \\
\hline \multirow[t]{2}{*}{43} & $0.329 \pm 0.02$ & \multirow[t]{2}{*}{245} & $0.324 \pm 0.01$ \\
\hline & $0.353 \pm 0.01$ & & $0.348 \pm 0.005$ \\
\hline \multirow[t]{2}{*}{11} & 0.333 & \multirow[t]{2}{*}{45} & $0.335 \pm ?$ \\
\hline & $0.361 \pm 0.008$ & & $0.360 \pm 0.002$ \\
\hline \multirow[t]{2}{*}{100} & $0.324 \pm 0.02$ & \multirow[t]{2}{*}{100} & $0.314 \pm 0.01$ \\
\hline & $0.340 \pm 0.01$ & & $0.338 \pm 0.007$ \\
\hline \multirow[t]{2}{*}{-100} & $0.348 \pm ?$ & \multirow[t]{2}{*}{-100} & $0.349 \pm ?$ \\
\hline & $0.398 \pm 0.03$ & & $0.401 \pm ?$ \\
\hline
\end{tabular}

TABLE II: The predicted value of $\alpha_{s}\left(M_{\tau}\right)$ in dependence of chosen values for the coefficients $d_{0}^{[1] 4}, d_{0}^{[1] 5}$. The second and the forth columns differ in the the number of terms in the perturbative series included. The upper value of $\alpha_{s}$ is the one predicted within FOPT, the lower corresponds to CIPT. The uncertainty in the value of $\alpha_{s}$ corresponds to changing the normalization point $\mu$ as follows $\mu^{2} / M_{\tau}^{2}=0.4-2$. The entry with question mark means that an equation for $\alpha_{s}(\mu)$ does not have a solution for some value of $\mu$ within the interval.

\begin{tabular}{|c|c|c|c|c|c|}
\hline Method & $\alpha_{s}\left(M_{\tau}\right)$ & $\Delta \delta_{P}^{\exp }$ & $\Delta \mu$ & $\Delta d_{0}^{[1] 4}$ & $\Delta d_{0}^{[1] 5}$ \\
\hline FOPT & $0.330 \pm 0.006 \pm 0.02$ & 0.006 & 0.019 & 0.0045 & 0.0011 \\
\hline CIPT & $0.354 \pm 0.009 \pm 0.006$ & 0.009 & 0.0036 & 0.0042 & 0.0019 \\
\hline
\end{tabular}

TABLE III: The value of $\alpha_{s}\left(M_{\tau}\right)$ obtained with $\delta_{P}^{\exp }, d_{0}^{[1] 4}$ and $d_{0}^{[1] 5}$ fixed to their central values according to eqs. (31, 18, 19) together with corresponding errors.

correspondingly. The difference between FOPT and CIPT decreases significantly, and this remains true even after extrapolating to $\alpha_{s}\left(M_{Z}\right)$.

$$
\begin{aligned}
\alpha_{s}^{\mathrm{FOPT}}(3 \mathrm{GeV}) & =0.263 \pm(0.013 \mid 0.014), \\
\alpha_{s}^{\mathrm{CIPT}}(3 \mathrm{GeV} & =0.265 \pm(0.005 \mid 0.008), \\
\alpha_{s}^{\mathrm{FOPT}}\left(M_{Z}\right) & =0.1198 \pm(0.002 \mid 0.003), \\
\alpha_{s}^{\mathrm{CIPT}}\left(M_{Z}\right) & =0.1203 \pm(0.009 \mid 0.0016) .
\end{aligned}
$$

The same coefficients $d_{0}^{[1] 4}$ and $d_{0}^{[1] 5}$ can be used to predict [16, 17] (the non-singlet part of) corrections of orders $\alpha_{s}^{4}$ and $\alpha_{s}^{5}$ to the $R$-ratio in $\mathrm{Z}$ decays:

$$
R\left(n_{f}=5\right)=1+a_{s}+1.409 a_{s}^{2}-12.77 a_{s}^{3}+(-97 \pm 44) a_{s}^{4}+(76 \pm 230) a_{s}^{5} .
$$

It is also of interest to display $R(s)$ for $n_{f}=3$ and 4 , which is accessible at $e^{+} e^{-}$colliders at lower energies.

$$
\begin{aligned}
& R\left(n_{f}=4\right)=1+a_{s}+1.525 a_{s}^{2}-11.52 a_{s}^{3}+(-112 \pm 30) a_{s}^{4}+(-245 \pm 160) a_{s}^{5} . \\
& R\left(n_{f}=3\right)=1+a_{s}+1.640 a_{s}^{2}-10.28 a_{s}^{3}+(-129 \pm 16) a_{s}^{4}+(-635 \pm 100) a_{s}^{5} .
\end{aligned}
$$

Our results are close to those of [16, 17], they employ, however, the additional information from [12, 31].

These formulae demonstrate rather good convergency for $n_{f}=5$ and a reasonably good one for $n_{f}=3$ and 4 if our predictions for the coefficients $d_{0}^{[1] 4}, d_{0}^{[1] 5}$ deviate from the true values within the assumed error margins. 


\begin{tabular}{|c|c|c|c|}
\hline$d_{0}^{[1] 4}$ & $\alpha_{s}^{4}$ & $d_{0}^{[1] 5}$ & $\alpha_{s}^{5}$ \\
\hline 27 & $0.256 \pm 0.007$ & 145 & $0.256 \pm 0.003$ \\
\cline { 2 - 2 } & $0.262 \pm 0.004$ & & $0.261 \pm 0.002$ \\
\hline \multirow{2}{*}{43} & $0.256 \pm 0.007$ & 245 & $0.254 \pm 0.003$ \\
\cline { 2 - 2 } & $0.26 \pm 0.004$ & & $0.259 \pm 0.002$ \\
\hline \multirow{2}{*}{11} & $0.257 \pm 0.006$ & \multirow{2}{*}{45} & $0.258 \pm 0.005$ \\
\cline { 2 - 2 } & $0.264 \pm 0.003$ & & $0.263 \pm 0.001$ \\
\hline \multirow{2}{*}{100} & $0.253 \pm 0.008$ & \multirow{2}{*}{100} & $0.249 \pm 0.004$ \\
\cline { 2 - 2 } & $0.255 \pm 0.008$ & & $0.254 \pm 0.002$ \\
\hline \multirow{2}{*}{-100} & $0.264 \pm 0.01$ & \multirow{2}{*}{100} & $0.277 \pm ?$ \\
\cline { 2 - 2 } & $0.279 \pm 0.02$ & & $0.28 \pm ?$ \\
\hline
\end{tabular}

TABLE IV: The predicted value of $\alpha_{s}\left(M_{\tau}\right)$ in dependence of chosen values for the coefficients $d_{0}^{[1] 4}, d_{0}^{[1] 5}$ for a hypothetical case of $M_{\tau}=3 \mathrm{GeV}$. The third and the forth columns differ in the the number of terms in the perturbative series included. The upper value of $\alpha_{s}$ is the one predicted within FOPT, the lower corresponds CIPT. The uncertainty in the value of $\alpha_{s}$ corresponds to changing the normalization point $\mu$ as follows $\mu^{2} / M_{\tau}^{2}=0.4-2$. The entry with question mark means that an equation for $\alpha_{s}\left(M_{\mu}\right)$ does not have a solution for some value of $\mu$ within the interval.

\section{B. Quadratic mass corrections}

Let us first discuss the function $D_{2}^{[1]}$. The FAC/PMS predictions can be easily obtained following [37; they are listed in Table $\mathrm{V}$.

\begin{tabular}{|c|l|l|l|l|}
\hline$n_{f}$ & $d_{3}^{\text {exact }}$ & $d_{3}^{\text {FAC }}$ & $d_{3}^{\text {PMS }}$ & $d_{4}^{\text {FAC } / \text { PMS }}$ \\
\hline \hline 3 & 107.5 & 89.86 & 91.17 & $1200 \pm 400$ \\
\hline 4 & 94.37 & 79.13 & 80.11 & $950 \pm 300$ \\
\hline 5 & 81.54 & 68.66 & 69.33 & $750 \pm 200$ \\
\hline
\end{tabular}

TABLE V: Estimations of the coefficients $d_{3}=d_{2}^{[1] 3}$ and $d_{4}=d_{2}^{[1] 4}$ in the function $D_{2}^{[1]}$ based on the FAC and PMS optimizations. The estimation of $d_{2}^{[1] 4}$ employs the exact value of $d_{2}^{[1] 3}$.

We again restore the $n_{f}$ dependence of the coefficient $d_{2}^{[1] 4}$ as predicted by FAC/PMS:

$$
d_{2}^{[1] 4}(\mathrm{FAC} / \mathrm{PMS})=1931.44-281.956 n_{f}+9.0294 n_{f}^{2}-0.0156289 n_{f}^{3}
$$

as well as that of $d_{2}^{[1] 3}$ :

$$
\begin{aligned}
& d_{2}^{[1] 3}(\mathrm{FAC})=123.654-11.6638 n_{f}+0.133293 n_{f}^{2}, \\
& d_{2}^{[1] 3}(\mathrm{PMS})=125.975-12.0028 n_{f}+0.134769 n_{f}^{2}
\end{aligned}
$$

to be compared with

$$
d_{2}^{[1] 3}(\text { exact })=148.978-14.3097 n_{f}+0.16463 n_{f}^{2}
$$

The comparison of estimates and exact results reveals a picture qualitatively similar to the massless case but with some modifications. A few important observations are in order.

1. All three terms of the $n_{f}$ expansion of $d_{2}^{[1] 3}$ are successfully predicted within about $20 \%$ accuracy.

2. Unlike the massless case the agreement between FAC/PMS predictions for the coefficient $d_{2}^{[1] 3}$ for $n_{f}=3,4,5$ and the corresponding exact numbers is within the range of $15 \%-20 \%$. On the other hand, the estimation of accuracy 
of the $\alpha_{s}^{3}$ fixed $n_{f}$ predictions obtained exclusively from the knowledge of the subleading contribution of $O\left(\alpha_{s}^{3} n_{f}\right)$ is of the right order of magnitude but somewhat less. All this is probably a consequence of a significantly larger $n_{f}$ independent contribution.

3. At $\mathcal{O}\left(\alpha_{s}^{4}\right)$ the exact result for the full coefficient

$$
d_{2}^{[1] 4}=d_{2,0}^{[1] 4}+d_{2,1}^{[1] 4} n_{f}+d_{2,2}^{[1] 4} n_{f}^{2}+d_{2,3}^{[1] 4} n_{f}^{3}
$$

is unknown, apart from its leading and subleading terms in $n_{f}$. The prediction for the subleading coefficient $d_{2,2}^{[1] 4}=9.0$ is by $50 \%$ larger than the exact value 6.07 . The predicted values for $d_{2,0}^{[1] 4}$ and $d_{2,1}^{[1] 4}$ are very large. In view of this largeness, the estimate $\left(\left.d_{2,2}^{[1] 4}\right|_{\text {exact }}-\left.d_{2,2}^{[1] 4}\right|_{F A C}\right) n_{f}^{2}\left(=30,50,80\right.$ for $n_{f}=3,4,5$ respectively) looks somewhat too optimistic. Therefore we assign a conservative $30 \%$ uncertainty to the fixed $n_{f}$ predictions listed in the fifth column of the Table $\mathrm{V}$.

\begin{tabular}{|c|c|c|c|}
\hline$n_{f}$ & $k_{3}^{\mathrm{FAC}}$ & $k_{3}^{\mathrm{PMS}}$ & $k_{4}^{\mathrm{PMS}}$ \\
3 & 199.1 & $200 \pm 60$ & $2200 \pm 1500$ \\
\hline 4 & 171.2 & $170 \pm 50$ & $1800 \pm 1100$ \\
\hline 5 & 144.7 & $145 \pm 40$ & $1400 \pm 900$ \\
\hline
\end{tabular}

TABLE VI: Estimates of the coefficients $k_{3}=k_{2}^{[2] 3}$ and $k_{4}=k_{2}^{[2] 4}$ in the function $\Pi_{2}^{[2]}$ based on the FAC and PMS optimizations. The estimate of $k_{2}^{[1] 4}$ employs the predicted value for $k_{3}$; the corresponding uncertainties include only the ones induced by $k_{3}$.

At last, we repeat the analysis for the function $\Pi_{2}^{[2]}$. The results of FAC/PMS optimization methods are given in Table VI. Using the values of $k_{3}^{\mathrm{PMS}}$ from the table for $n_{f}=3,4,5$ we reconstruct the corresponding full $n_{f}$ dependence:

$$
k_{2}^{[1] 3}=294.472-33.2429 n_{f}+0.696598 n_{f}^{2} .
$$

The comparison with the known terms of order $n_{f}$ and $n_{f}^{2}\left(-32.0843 n_{f}, 0.464663 n f^{2}\right)$ demonstrates a remarkably good agreement for the subleading $n_{f}$ contribution. The $50 \%$ error in the predicted value of the $n_{f}^{2}$ contribution looks natural as the corresponding coefficient is small. Following the same line of reasoning as above we have assigned a $30 \%$ uncertainty to the $O\left(\alpha_{s}^{3}\right)$ fixed $n_{f}$ result.

To get a general idea about the size of the $\alpha_{s}^{4}$ contribution to eq. (14) we used FAC/PMS and the predicted $\alpha_{s}^{3}$ coefficient. The results are listed in the forth column of Table VI.

Let us now consider the effect of $\alpha_{s}^{3}$ and $\alpha_{s}^{4}$ corrections on the determination of the strange quark mass 41. The mass correction to the $R_{\tau}$ depend on both functions $D_{2}^{[1]}$ and $\Pi_{2}^{[2]}$. Let us use the central ALEPH value of $\alpha_{s}\left(M_{\tau}\right)=0.334$ when estimating the size of the perturbative corrections. For fixed order one finds the mass correction to the total rate:

$$
\begin{aligned}
\delta_{u s, 2}^{00} & =-8 \frac{m_{s}^{2}}{M_{\tau}^{2}}\left(1 .+5.33 a_{s}+46.0 a_{s}^{2}+284 a_{s}^{3}\right. \\
& \left.+0.75 a_{s}^{3} k_{2}^{[2] 3}+a_{s}^{4}\left(723 .+0.25 d_{2}^{[1] 4}+9.84 k_{2}^{[2] 3}+0.75 k_{2}^{[2] 4}\right)\right) \\
& =-8 \frac{m_{s}^{2}}{M_{\tau}^{2}}(1 .+0.567+0.520+0.521 \pm 0.05+0.593) \\
& =-8 \frac{m_{s}^{2}}{M_{\tau}^{2}}(3.2 \pm 0.6),
\end{aligned}
$$

where in the last line we have assumed the (maximal!) value of the $\mathcal{O}\left(\alpha_{s}^{4}\right)$ term as an estimate of the theoretical uncertainty (this convention will be used also below). 
For the "contour improved" series one obtains

$$
\begin{aligned}
\tilde{\delta}_{u s, 2}^{00}=-8 \frac{m_{s}^{2}}{M_{\tau}^{2}}\left(1.44+3.65 a_{s}\right. & +30.9 a_{s}^{2}+72.2 a_{s}^{3}+1.18 a_{s}^{3} k_{2}^{[2], 3} \\
& \left.+a_{s}^{4}\left(0.678 d_{2}^{[1] 4}+1.06 k_{2}^{[2] 4}\right)\right) \\
= & -8 \frac{m_{s}^{2}}{M_{\tau}^{2}}(1.44+0.389+0.349+0.371 \pm 0.09+0.403) \\
= & -8 \frac{m_{s}^{2}}{M_{\tau}^{2}}(2.95 \pm 0.4) .
\end{aligned}
$$

Now we consider the contributions of spin 1 and spin 0 separately. The lowest moments $(L=0)$ of the spindependent functions depend on a nonperturbative quantity and, thus, can not be treated perturbatively in principle [7].

For the spin one part and for $(k, l)=(0,1)$ we find

$$
\begin{aligned}
\delta_{u s, 2}^{(1) 01} & \left.=-5 \frac{m_{s}^{2}}{M_{\tau}^{2}}\left(1 .+4.83 a+35.7 a_{s}^{2}+276 . a_{s}^{3}+a_{s}^{4}\left(1350+d_{2}^{[1] 4}\right)\right)\right) \\
& =-5 \frac{m_{s}^{2}}{M_{\tau}^{2}}(1 .+0.514+0.404+0.331+0.326) \\
& =-5 \frac{m_{s}^{2}}{M_{\tau}^{2}}(2.58 \pm 0.33)
\end{aligned}
$$

and

$$
\begin{aligned}
\tilde{\delta}_{u s, 2}^{(1) 01} & =-5 \frac{m_{s}^{2}}{M_{\tau}^{2}}\left(1.37+2.55 a+16.1 a_{s}^{2}+135 a_{s}^{3}+0.895 a_{s}^{4} d_{2}^{[1] 4}\right) \\
& =-5 \frac{m_{s}^{2}}{M_{\tau}^{2}}(1.37+0.271+0.182+0.163+0.137) \\
& =-5 \frac{m_{s}^{2}}{M_{\tau}^{2}}(2.12 \pm 0.14) .
\end{aligned}
$$

Note that spin 1 contribution is determined by the component $\Pi^{[1]}$ alone and is known up to third order. Clearly, this series is decreasing in a reasonable way (comparable to the behaviour of $\tilde{\delta}_{u s, 2}^{00}$ ) and, at the same time, only moderately dependent on the improvement prescription with $\tilde{\delta}_{u s, 2}^{(1) 01} / \delta_{u s, 2}^{(1) 01}=0.82$. On the basis of Eq. (48) this moment might well serve for a reliable $m_{s}$ determination, with a sufficiently careful interpretation of the theoretical uncertainty.

The corresponding spin zero part is, per se, proportional to $m_{s}^{2}$ (not counting non-perturbative, so-called "condensate" contributions) and thus could be considered as ideal for a measurement of $m_{s}$. However, the behaviour of the perturbative series

$$
\begin{aligned}
\delta_{u s, 2}^{(0) 01}= & \frac{3}{2} \frac{m_{s}^{2}}{M_{\tau}^{2}}\left(1 .+9.33 a+110 a_{s}^{2}+1323 a_{s}^{3}\right. \\
& +a_{s}^{4}\left(12200+d_{2}^{[1] 4}+17.5 k_{2}^{[2] 3}\right) \\
= & \frac{m_{s}^{2}}{M_{\tau}^{2}}(1 .+0.992+1.24+1.59+2.16) \\
= & \frac{3}{2} \frac{m_{s}^{2}}{M_{\tau}^{2}}(7.0 \pm 2)
\end{aligned}
$$

and

$$
\begin{aligned}
\tilde{\delta}_{u s, 2}^{(0) 01}= & \frac{3}{2} \frac{m_{s}^{2}}{M_{\tau}^{2}}\left(3.19+11.2 a+126 . a_{s}^{2}+289 . a_{s}^{3}+6.63 a_{s}^{3} k_{2}^{[2] 3}\right. \\
& \left.+a_{s}^{4}\left(2.71 d_{2}^{[1] 4}+7.76 k_{2}^{[2] 4}\right)\right) \\
= & \frac{3}{2} \frac{m_{s}^{2}}{M_{\tau}^{2}}(3.19+1.19+1.42+1.94+2.6 \pm 1.31) \\
= & \frac{3}{2} \frac{m_{s}^{2}}{M_{\tau}^{2}}(10.3 \pm 2.6)
\end{aligned}
$$


shows a rapid growth of the coefficients. The series is not expected to provide an accurate prediction for the mass effects.

\section{SUMMARY}

Implications of the newly calculated $\alpha_{s}^{4} n_{f}^{2}$ terms together with the $\alpha_{s}^{4} n_{f}^{3}$ terms for an improved extraction of $\alpha_{s}$ from the $\tau$ decay are presented. Arguments are presented in support of predictions for the remaining terms of order $\alpha_{s}^{4} n_{f}$ and $\alpha_{s}^{4} n_{f}^{0}$ which are based on FAC or PMS optimization. The complete calculation will lead to a reduction of the theory uncertainty within the frameworks of FOPT or CIPT down to a negligible amount. However, an irreducible difference between the results from these two schemes of $\delta \alpha_{s}\left(M_{\tau}\right) \approx 0.02$ corresponding to $\delta \alpha_{s}\left(M_{Z}\right) \approx 0.002$ persists even after inclusion of $\mathcal{O}\left(\alpha_{s}^{4}\right)$ (and even $\mathcal{O}\left(\alpha_{s}^{5}\right)$ terms). Similar investigations, based on data up to higher energies (e. g. for fictitious heavy lepton of $3 \mathrm{GeV}$ of for sum rules based on $e^{+} e^{-}$data) would lead to significantly smaller errors.

New contributions of orders $\mathcal{O}\left(m_{s}^{2} \alpha_{s}^{4} n_{f}^{2}\right)$ and $\mathcal{O}\left(\alpha_{s}^{4} n_{f}^{2}\right)$ to (axial)vector correlators relevant for the QCD description of the semileptonic $\tau$ decay into hadrons are obtained. The moments $R^{(0,0)}$ and $R^{(0,1)}$ are evaluated separately for spin zero and spin one final states [7]. The results are tested against predictions of FAC/PMS optimization methods. Good agreement is found. This has motivated us to take the full of FAC/PMS predictions as the basis for a new extraction of $\alpha_{s}$ and $m_{s}$ from $\tau$-decays with $\mathcal{O}\left(\alpha_{s}^{4}\right)$ accuracy. Using $\delta_{P}^{\exp }=0.207$ we find 0.330 and 0.1192 for $\alpha_{s}^{\mathrm{FOPT}}\left(M_{\tau}\right)$ and $\alpha_{s}^{\mathrm{FOPT}}\left(M_{Z}\right)$ respectively. In the framework of contour improved evaluation these values increase to 0.354 and 0.1219 respectively

In contrast to the massless result, the PT series contributing to the $m_{s}^{2}$ dependent part seem to be barely convergent and the additional higher order terms seemingly do not lead to any significant improvement of the theoretical accuracy in the determination of the strange quark mass from the $\tau$ decays.

A slightly more favorable pattern of convergence is observed for the moments of the spin one contribution separately.

\section{ACKNOWLEDGMENTS}

The authors are grateful to A.A. Pivovarov for a lot of useful discussions. One of us (K.G. Ch.) acknowledges useful correspondence to K. Maltman and A.L. Kataev. This work was supported by the DFG-Forschergruppe "Quantenfeldtheorie, Computeralgebra und Monte-Carlo-Simulation" (contract FOR 264/2-1), by INTAS (grant 0000313), by RFBR (grant 01-02-16171), by Volkswagen Foundation and by the European Union under contract HPRNCT-2000-00149.

[1] R. Barate et al. (The ALEPH collaboration), Z. Phys. C 76, 15 (1997); Eur. Phys. J. C 4, 409 (1998); Eur. Phys. J. C 11, 599 (1999).

[2] K. Ackerstaff et al. (The OPAL collaboration), Eur. Phys. J. C 7, 571 (1999).

[3] K.G. Chetyrkin, J.H. Kühn, and A. Kwiatkowski, Phys. Rep. 277, 189 (1996).

[4] K. Maltman, Phys. Rev. D 58, 093015 (1998).

[5] K.G. Chetyrkin, and A. Kwiatkowski, Z. Phys. C 59, 5251993.

[6] K.G. Chetyrkin, and A. Kwiatkowski, hep-ph/9805232.

[7] K.G. Chetyrkin, J.H. Kühn, and A.A. Pivovarov, Nucl. Phys. B 533, 473 (1998).

[8] A. Pich and J. Prades, JHEP 9806, 013 (1998); ibid 9910, 004 (1999).

[9] J.G. Körner, F. Krajewski, and A.A. Pivovarov, Eur. Phys. J. C 20, 259 (2001);

[10] S. Chen, M. Davier, E. Gamiz, A. Hocker, A. Pich, and J. Prades, Eur. Phys. J. C 22, 31 (2001).

[11] K. Maltman, hep-ph/0209091.

[12] P.A. Baikov, K.G. Chetyrkin, and J.H. Kühn, Phys. Rev. Lett. 88, 012001 (2002).

[13] P.M. Stevenson, Phys. Rev. D 23, 2916 (1981); Phys. Lett. B 100, 61 (1981); Nucl. Phys. B 203, 472 (1982); Phys. Lett. B 231, 65 (1984).

[14] G. Grunberg, Phys. Lett. B 95, 70 (1980); Phys. Rev. D 29, 2315 (1984).

[15] N.V. Krasnikov, Nucl. Phys. B 192, 497 (1981);

[16] A.L. Kataev and V.V. Starshenko, Mod. Phys. Lett. A 10, 235 (1995).

[17] A.L. Kataev and V.V. Starshenko, Nucl. Phys. Proc. Suppl. 39BC, 312 (1995), arXiv:hep-ph/9408395.

[18] A.A. Pivovarov, Sov. J. Nucl. Phys. 54, 676 (1991); Z. Phys. C 53, 461 (1992); Nuovo Cim. 105A, 813 (1992).

[19] F. Le Diberder and A. Pich, Phys. Lett. B 286, 147 (1992); 289, 165 (1992).

[20] K. Schilcher, M.D. Tran, Phys. Rev. D 29, 570 (1984).

[21] E. Braaten, Phys. Rev. Lett. 53, 1606 (1988). 
[22] S. Narison, A. Pich, Phys. Lett. B 211, 183 (1988).

[23] E. Braaten, Phys. Rev. D 39, 1458 (1989).

[24] E. Braaten, S. Narison, A. Pich, Nucl. Phys. B 373, 581 (1992).

[25] P.A. Baikov, Phys. Lett. B 385, 404 (1996); Nucl. Inst. Meth. A389, 347 (1997);

P.A. Baikov and M. Steinhauser, Comp. Phys. Commun. 115, 161 (1998).

[26] P.A. Baikov, Phys. Lett. B 474, 385 (2000).

[27] D. Fliegner, A. Retey, and J.A. Vermaseren, hep-ph/0007221.

[28] J.A.M. Vermaseren, math-ph/0010025.

[29] G. 't Hooft, Nucl. Phys. B 61, 455 (1973).

[30] W.A. Bardeen, A.J. Buras, D.W. Duke and T. Muta, Phys. Rev. D 18, 3998 (1978).

[31] T. van Ritbergen, J.A. Vermaseren, and S.A. Larin, Phys. Lett. B 400, 379 (1997).

[32] K. Hagiwara et al., Phys. Rev. D 66, 010001 (2002).

[33] W.J. Marciano and A. Sirlin, Phys. Rev. Lett. 61, 1815 (1988).

[34] E. Braaten and C.S. Li, Phys. Rev. D 42, 3888 (1990).

[35] S. Groote, J.G. Korner and A.A. Pivovarov, Mod. Phys. Lett. A 13, 637 (1998).

[36] J.G. Körner, F. Krajewski, and A.A. Pivovarov, Phys. Rev. D 63, 036001 (2001).

[37] K.G. Chetyrkin, B.A. Kniehl, and A. Sirlin, Phys. Lett. B 402, 359 (1997).

[38] We thank Matthias Steihnauser and Robert Harlander for a useful discusson of this point.

[39] According to eq. (31) the latter includes (small) uncertainties in the values of $\left|V_{u d}\right|$ and the nonperturbative correction $\delta_{N P}$ in addition to the experimental error per se as displayed in eq. (30).

[40] This is in agreement to the analysis of Ref. [35], where it has been concluded that "the resummed values of $\alpha_{s}$ from $\tau$ decay lie outside the convergence radii and can therefore not be obtained from a power series expansion. Regular perturbation series do not converge to their resummed counterparts. The experimental value of $R_{\tau}$ appears to be too large for a fixed order perturbation analysis to apply". See also [36].

[41] For a recent review of various attempts to extract the strange quark mass from $\tau$ data see Ref. [1]]. 